\title{
Un modelo de gestión de inventarios basado en estrategia competitiva
}

\author{
An inventory management model based on competitive strategy \\ Adolfo González ${ }^{1 *}$ \\ Recibido 15 de noviembre de 2017, aceptado 17 de septiembre de 2018 \\ Received: November 15, 2017 Accepted: September 17, 2018
}

\begin{abstract}
RESUMEN
Este artículo presenta una metodología para la gestión de los inventarios, orientada a la estrategia competitiva de la empresa en ambientes multiproducto y con variabilidad en la demanda. La metodología propuesta se estructura en cuatro etapas: La primera, identifica y/o sitúa la estrategia de la empresa en un ambiente competitivo en relación a los niveles de servicio. La segunda etapa, establece una clasificación de los productos de acuerdo a criterios concordantes con la estrategia de la empresa, clasificando los productos en más y menos relevantes, en función de la demanda. En la tercera etapa, se realiza un pronóstico de la demanda, donde se emplea el coeficiente de variación como medida de variabilidad y como técnicas de pronóstico la suavización exponencial, aunque la metodología es flexible y se pueden emplear otros métodos de series de tiempo. La cuarta etapa, se orienta a la selección de una política de inventario acorde con las necesidades de la estrategia competitiva de la empresa como es la política de revisión periódica. La metodología de cuatro pasos, se implementó en una empresa líder en Chile del rubro de pernería y tornillería.
\end{abstract}

Palabras clave: Estrategia competitiva, gestión de inventarios, clasificación abc, pronóstico de la demanda, políticas de inventario.

\begin{abstract}
This article presents a methodology for inventory management, aimed at the competitive strategy of a company in multi-environments and variability in demand. The proposed methodology is divided into four stages: First, it identifies and locates the strategy of the company in a competitive environment in relation to service levels. The second stage provides a classification of products according to criteria consistent with company strategy, classifying products according to their relevance, depending on demand. In the third stage, the demand forecast is calculated using the coefficient of variation as a measure of variability and the forecasting technique is exponential smoothing, although the methodology is flexible and can be used other methods of time series. The fourth stage is focused on the selection of an inventory policy according to the needs of the competitive strategy of the company in this case it is used to periodic review policy. This four-step methodology was implemented in a leading company for the category of screws and bolts in Chile.
\end{abstract}

Keywords: Competitive strategy, inventory management, $A B C$ classification, demand forecasting, Inventory policies.

\footnotetext{
1 Magister en Ingeniería Industrial, Universidad Nacional Andrés Bello, Sazié 2320, Santiago, Región Metropolitana, Chile.

* Autor de correspondencia: adolfo.rgc@gmail.com
} 


\section{INTRODUCCIÓN}

La gestión de inventarios en una empresa, es una actividad interrelacionada con la cadena de valor de ésta y debe estar en concordancia con la estrategia y tácticas de la empresa, con el propósito de satisfacer a los clientes [1].

En ambientes competitivos, las empresas definen estrategias con el objetivo de alcanzar mayores cuotas de mercado para aumentar los márgenes de ganancias, como lo plantea Porter [2], desde esta visión y entendiendo que las inversiones en inventarios pueden llegar a ser significativas en los balances de las empresas y en consecuencia el manejo de los inventarios con metodologías orientadas a mantenerlos controlados, en función de los costos y rotaciones, pasa a ser primordial, pero, esta visión es meramente financiera y deja de lado las necesidades de los clientes [3]. En relación a esta perspectiva, las empresas deben utilizar una metodología que permita estimar las políticas de control de inventarios a lo largo de su cadena de valor [4].

Este trabajo se orienta al diseño y validación de un modelo que comienza con un análisis de la estrategia del negocio, para luego definir las clasificaciones de los inventarios. Con estos elementos se procede a la proyección de demandas, confluyendo en la selección e implementación de una política de inventarios. La característica principal de este modelo es la aplicabilidad y adaptación a los cambios de la demanda, pues, puede realizarse los pasos de manera cíclica y repetitiva, para así poder adaptarse constantemente a la estrategia de la empresa, la cual responde a un entorno competitivo.

\section{MARCO REFERENCIAL}

\section{Gestión de Inventario y Estrategia Competitiva}

La gestión de inventarios en función del nivel de servicio es una actividad que se relaciona directamente con la cadena de valor de la empresa [2] y está en concordancia con la estrategia y tácticas de la empresa, con el propósito de satisfacer a los clientes. La necesidad de crecimiento de las empresas que se encuentran en ambientes altamente competitivos, bajo estrategias de diferenciación por costos y que participan en la Industria de Pernería \& Tornillería en Chile, impulsan la necesidad de diferenciarse, en este sentido, el implementar estrategias de Océano Azul [5], entrega una perspectiva de estrategia agregada a las empresas. En este contexto, la identificación de los elementos diferenciadores relacionados con la estrategia de diferenciación, indican que el manejo del nivel de servicio, es un eje central para que la estrategia de diferenciación definida se aplique efectivamente. En consecuencia, en primera instancia, orientándose a obtener un manejo controlado de la operación logística de la empresa, en función de nivel de servicio, se identifican las directivas que conformarán la clasificación de inventario.

\section{Clasificación de Inventarios}

En relación a la clasificación de inventarios de productos, existen diferentes trabajos donde se utilizan aproximaciones de modelamiento matemático para establecer las diferentes agrupaciones de productos utilizando una metodología ABC. Algunos modelos emplean metodología basada en conjuntos difusos para la clasificación y control de inventario llamado ABC-fuzzy clasificación (ABCFC). Otra variación de clasificación donde se usan metodologías basadas en múltiples criterios. En este sentido, es posible identificar diversas formas de aplicar las clasificaciones de productos, teniendo como objetivo una forma específica de aplicarlo que responda a las necesidades del negocio [1]. El trabajo de Méndez \& López [1], deja en evidencia la posibilidad de utilizar y adaptar un modelo de clasificación de productos a las necesidades del negocio para el cual se utilice.

\section{Pronóstico de la Demanda}

En tercera instancia, manteniendo la orientación a obtener un manejo controlado de la operación logística de la empresa, entendiendo que gestionar inventarios es un tema complejo, pues, se observa que hay demasiado de lo que no se vende o consume, y productos agotados de lo que sí se vende, esto como un reflejo del desconocimiento de la demanda. Bajo este contexto el pronosticar la demanda, conlleva a la estimación anticipada del valor de una variable, la cual es una piedra angular para la toma de decisiones dentro de la empresa que trasciende al ámbito logístico [6]. En este contexto se observa que los modelos de pronóstico de la demanda utilizados con más frecuencia son [7]; Método Promedio Simple, Método Promedio Móvil Simple, Método de Promedios Ponderados Móviles, Método de Regresión Lineal, Suavizamiento Exponencial 
Simple, Método de Índices de Estacionalidad y Suavizamiento Exponencial Doble [8].

En este sentido, el criterio a seguir para establecer un pronóstico con base en la información disponible y características de la demanda, está relacionado con el enfoque de tiempo, donde se distinguen; los de corto, mediano y largo plazo, por tanto, los modelos aplicados deben evaluarse para determinar el más adecuado en función de la demanda del producto analizado $[9,10]$.

\section{Políticas de Inventario}

La cuarta instancia, orientada a la obtención del manejo controlado de la operación logística de la empresa, corresponde a la elección de la política de inventario o método de gestión de inventario a utilizar. En este contexto, se observan métodos posibles de usar para un sistema de gestión de inventarios que se adecue con el comportamiento de la demanda ya analizados en instancia anteriores. Se debe tener en cuenta los modelos o también denominado políticas de inventario, se pueden clasificar en tres grupos principales, los cuales son;

Determinísticos; los cuales poseen muchas restricciones y se basan en el modelo de Wilson del Lote Económico de compra (EOQ).

Demanda conocida. No tiene variaciones en el tiempo [6].

Probabilístico; los cuales integran fluctuaciones aleatorias de la demanda. Estos sistemas se clasifican a su vez según el tipo de demanda, ya sean dependientes o independientes [6].

La selección de la política de inventarios, se debe orientar a responder la pregunta orientada a establecer cada cuánto tiempo debe revisarse el inventario, cuándo ordenar y cuánto ordenar. No obstante, se debe considerar que puede existir una variación significativa si se consideran los aspectos relacionados con el tipo de producto (terminado o materia prima) y el ambiente de producción.

El trabajo investigativo de Gutiérrez \& Vidal presenta la selección y aplicación de modelos que resuelven de manera práctica el problema de selección de las políticas de inventario, el cual entrega una directriz estimativa a considerar [4].

\section{APLICACIÓN DEL MODELO}

\section{Identificación de la Estrategia competitiva de la Empresa}

La empresa definió su estrategia de negocios con base en el modelo de negocios, que es el más utilizado por las empresas participantes de la Industria de Pernería \& Tornillería en Chile, denominada; Estrategia de diferenciación por costos (Liderazgo en Costos). En este sentido, los impulsores de valor (Drivers) que se siguen son; la tasa de crecimiento de las ventas, margen de beneficio de explotación, inversión en capital circulante, inversión en capital fijo y costo del capital [2].

El vector principal de la estrategia de la empresa, dado como un elemento diferenciador, según la estrategia de diferenciación específica de la empresa (Océano Azul) [5], está dada por el nivel de servicio (inventario) y representa la probabilidad esperada de no llegar a una situación de falta de existencias. Esto implica que para el mix de productos que la empresa posee, se asegura al cliente, que encontrará los productos que seleccione para adquirir del mix de productos que la empresa expone.

Bajo este contexto, el indicador de nivel de servicio, está dado por; los productos comprometidos de entrega en una nota de venta, versus los entregados al cliente, por tanto, las rebajas a las notas de venta son considerados una baja en el nivel de servicio, en este lineamiento, la empresa definió un nivel de servicio del 98\%, como cota inferior (Mínimo), pues en base a la estrategia definida con este parámetro se alcanza la diferenciación en el mercado. Bajo este contexto, la empresa no considera la organización de los inventarios por el criterio de la importancia económica, ni el manejo de los espacios en bodega, como elementos a considerar, pues define absorber los impactos de estos aspectos en la operación de la empresa, privilegiando el elemento de existencia de productos, el que define como la rotación del producto que representa las veces que el producto es vendido en un periodo de tiempo.

Este nivel de servicio establecido por la empresa, está definido como un porcentaje de entrega de productos al cliente, dado por (1);

$$
N_{s}=\frac{P_{F}}{P_{N V}} \times 100
$$


Donde;

$\mathbf{N}_{\mathbf{S}}$ : Porcentaje del Nivel de Servicio, por nota de venta.

$\mathbf{P}_{\mathbf{F}}$ : Número de SKU, Entregado efectivamente al Cliente en Nota de Venta.

$\mathbf{P}_{\mathrm{NV}}$ : Número de SKU, comprometidos a entregar al Cliente en Nota de Venta.

\section{Clasificación de los Inventarios de la Empresa}

Con base en los lineamientos dados por la empresa en función de su estrategia competitiva, se realiza la clasificación de inventario.

En la realización de la clasificación se consideran criterios globales, como:

Criterio A: La cantidad de movimiento (rotación) que presenta el producto (SKU), apuntando a suavizar los efectos de la alta variabilidad de la demanda, este criterio es denominado por movimiento [1].

Criterio B: La Importancia, el cual se considera como un criterio compuesto, apuntando al manejo de los costos de inventarios, o sea, relaciona los costos y rotaciones, de manera de tener un criterio económico de clasificación [1].

Criterio C: El Acopio, el cual se considera como un criterio compuesto, apuntando al manejo de los espacios en las bodegas, o sea, es una relación de carácter físico, apuntando a racionalizar los espacios de bodegaje de la empresa [1].

Tomando en consideración la estrategia de la empresa, donde el elemento a considerar está dado por el nivel de servicio y descarta los criterios de Importancia y Acopio, se define utilizar solo el criterio de cantidad, denominado Criterio A.

Bajo la directriz del criterio A, la clasificación esta estratificada en; clasificación A, que representa el $2,5 \%$ de los productos que generan el $80 \%$ de la rotación, la clasificación B, que representa el 7,3\% de los productos que generan el $15 \%$ de la rotación, la clasificación C, que representa el 52,2\% de los productos que generan el $5 \%$ de la rotación y la clasificación D, que representa el $37,9 \%$ de los productos que generan el $0 \%$ de la rotación.

La Tabla 1, expone el resultado de la clasificación utilizando el criterio seleccionado.
Tabla 1. Clasificación de productos por rotación.

\begin{tabular}{|c|c|c|}
\hline Clasificación & $\mathbf{N}^{\mathbf{0}}$ Productos & \% Rotación \\
\hline A & 476 & $80 \%$ \\
\hline B & 1.397 & $15 \%$ \\
\hline C & 9.933 & $5 \%$ \\
\hline D & 7.219 & $0 \%$ \\
\hline Total & 19.025 & \\
\hline
\end{tabular}

Las acciones definidas a realizar para cada clasificación son;

Clasificación A, Realización de un estricto control, con revisiones periódicas en relación a los niveles de existencias con atención en la exactitud de los registros.

Clasificación B, Realización de un control intermedio, con revisiones continúas en relación a los niveles de existencias con atención en el registro de los datos.

Clasificación C, Realización de un control menos rígido, con revisiones continuas en relación a los niveles de existencias con atención en el registro de los datos.

Clasificación D, Realización de un análisis de estos productos, para determinar; darlos de baja o reimpulsar su venta.

\section{Pronóstico de la Demanda de los Productos de la Empresa}

A los productos clasificados como A, B y C, se les procedió a pronosticar la demanda, utilizando las técnicas de Método Promedio Simple, Método Promedio Móvil Simple, Método de Promedios Ponderados Móviles, Suavizamiento Exponencial Simple y Suavizamiento Exponencial Doble [7, 8].

El pronóstico de la demanda se realiza para cada producto realizando un análisis del comportamiento histórico a 12 meses históricos, a cada producto se le aplica el Método de Promedio Simple, Método de Promedio Móvil Simple, Método de Promedios Ponderados Móviles, Suavizamiento Exponencial Simple y Suavizamiento Exponencial Doble.

Posteriormente para seleccionar el modelo de pronóstico más adecuado al comportamiento histórico de la demanda del producto, se calculan para modelo de pronóstico la desviación estándar 
absoluta, el error de pronóstico acumulado, el error porcentual absoluto medio y la señal de rastreo [12].

Mediante la utilización de la suma acumulativa de los errores para el sesgo y la desviación media absoluta para la dispersión. Teniendo en cuenta que la empresa asigna la misma ponderación a los errores de sesgo y a los errores de dispersión, se procedió a seleccionar el método de pronóstico que representa de mejor manera a la variabilidad de la demanda y permiten ajustar de mejor manera los inventarios en función de los lineamientos de la estrategia de la empresa [7, 9].

Un criterio importante que se ha definido en la estrategia competitiva de la empresa, con respecto a los inventarios, es la existencia de tres meses de inventarios por rotación mensual, o sea, cantidad de rotación mensual multiplicado por tres meses, corresponde a la existencia máxima en la bodega de venta y para el caso de la existencia en bodega de importaciones un periodo de seis meses [10].

El ciclo de cálculo de la demanda para cada producto es revisado en forma periódica en función de la clasificación de los productos, desde clasificación A hasta la $\mathrm{C}$, pues, la variabilidad de la demanda provocada por acciones comerciales, condiciones del mercado o cambios en la estrategia de la empresa, provocan cambios en los stocks que afectan el nivel de servicio.

\section{Identificación de Política de Inventario para la Empresa}

El análisis de la demanda entrega lineamientos en función de la variabilidad de ésta y el carácter estacional, basado en esto, en conjunto con la clasificación dada, se procede a seleccionar las políticas de inventario para las clasificaciones A, B y C $[4,12]$.

Para la clasificación A; se define utilizar una política de revisión periódica, pues esta política contiene un criterio de control más estricto acorde con la definición de la clasificación de productos [13, 14].

Para la clasificación B y C; se define utilizar una política de revisión continua, pues esta política contiene criterios de control menos estrictos acorde con la definición de la clasificación [4].

\section{RESULTADOS}

La aplicación del modelo, a los grupos de productos clasificados como A, B y C, por un periodo de tres meses, impulsó el crecimiento del nivel de servicio, hacia el $98 \%$ definido en la estrategia de la empresa.

La Tabla 2, expone los resultados obtenidos en relación a los niveles de servicio antes y después de la aplicación del modelo, indicando además el nivel servicio definido de un $98 \%$, el cual es concordante con la estrategia definida por la empresa para cada clasificación de productos.

Los niveles de servicio alcanzados después de la aplicación del modelo para las clasificaciones de productos A y B, superan el nivel de servicio del 98\% definido por la empresa.

En los productos clasificados como C, se observó que el nivel de servicio no alcanzó o superó el 98\% de nivel de servicio definido por la empresa, pero se aprecia un incremento del $18,43 \%$, respecto a los niveles de servicio de esta clasificación antes de aplicar el modelo.

Relacionando el porcentaje de participación de la rotación de cada clasificación de productos de la Tabla 1, con los niveles de servicio obtenidos antes y después de la aplicación del modelo, expuestos en la Tabla 2. Se construye la Tabla 3, que muestra el aporte de los niveles de servicio de cada clasificación

Tabla 2. Cambio Niveles de Servicio.

\begin{tabular}{|c|c|c|c|}
\hline Clasificación & $\begin{array}{c}\text { \% Nivel } \\
\text { Servicio } \\
\text { Antes }\end{array}$ & $\begin{array}{c}\text { \% Nivel } \\
\text { Servicio } \\
\text { Después }\end{array}$ & $\begin{array}{c}\text { \% Nivel } \\
\text { Servicio } \\
\text { Definido }\end{array}$ \\
\hline A & $88,02 \%$ & $99,75 \%$ & $98 \%$ \\
\hline B & $79,12 \%$ & $99,67 \%$ & $98 \%$ \\
\hline C & $40,82 \%$ & $59,31 \%$ & $98 \%$ \\
\hline
\end{tabular}

Tabla 3. Nivel de Servicio en relación a la clasificación de los productos.

\begin{tabular}{|l|c|c|c|}
\hline Clasificación & \% Rotación & Antes & Después \\
\hline A & $80 \%$ & $70,43 \%$ & $79,81 \%$ \\
\hline B & $15 \%$ & $11,87 \%$ & $14,95 \%$ \\
\hline C & $5 \%$ & $2,04 \%$ & $2,96 \%$ \\
\hline Total & $100 \%$ & $84,33 \%$ & $97,72 \%$ \\
\hline
\end{tabular}


al nivel de servicio total de la empresa para con el cliente.

El aumento en el nivel de servicio de la empresa después de aplicado el modelo es de 13,39\%.

\section{CONCLUSIONES}

El modelo aplicado posee cuatro etapas, donde cada etapa es iterativa en sí misma, de manera de realizar cambios en función de; mejorar la forma de utilizar el lineamiento establecido en la implementación, o por cambios de una etapa anterior que afecta a la implementación establecida y que se encuentra operando.

El modelo expuesto, es del tipo secuencial y el impacto de una etapa anterior en una etapa siguiente es significativo, por tanto, si una etapa anterior realiza algún cambio, es necesario revisar las etapas siguientes y adaptar la implementación existente. Así, si por ejemplo cambia algún elemento de la estrategia competitiva en la etapa uno, se deberá evaluar en las etapas subsiguientes el impacto que este cambio genera y proceder al ajuste de la implementación en relación al cambio.

La aplicación de la etapa uno, en relación a la estrategia de la empresa, permite establecer en primera instancia los drivers de la estrategia y los elementos diferenciadores de la estrategia de la empresa, pues así, como en este caso aplicado, el nivel de servicio en función de las rotaciones, dejando de lado los parámetros de espacio y costos. Se entiende que el aplicar este paso en otra empresa, este elemento puede cambiar, pues la implementación no necesariamente puede ser la misma a la expuesta en este caso aplicado.

La etapa dos, toma los lineamientos de la etapa uno para realizar la clasificación de inventario en función de la estrategia de la empresa, por tanto, la clasificación de los productos no está relacionada netamente por los atributos de los productos (costo, peso, volumen, etc.), en este sentido los atributos se seleccionan en relación directa o significativa con la estrategia de la empresa.

En relación al caso expuesto, se aprecia que la clasificación en función de las rotaciones, expone la clasificación D (Tabla 1), que implica que los productos clasificados en esta categoría, deben ser evaluados, para ser dados de baja. Un aspecto relevante de la clasificación, apunta a poner atención en los productos que más relevancia tienen para la estrategia competitiva de la empresa.

Desde una mirada global, la aplicación de esta etapa, permite realizar acciones sobre los inventarios de la

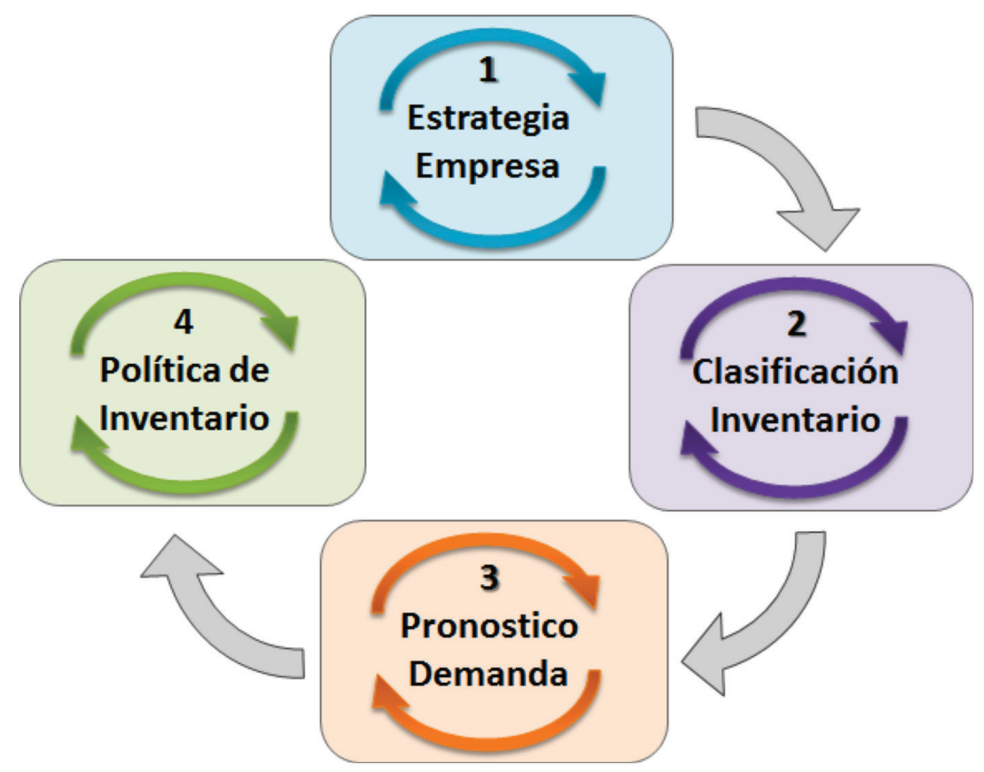

Figura 1. Gestión de inventarios basado en estrategia competitiva. 
empresa apuntado al nivel de servicio, pues permite focalizar los esfuerzos en los productos clasificados como relevantes para la estrategia de la empresa.

La etapa tres, relacionada con el pronóstico de la demanda permite obtener una proyección de necesidades de productos y estimar las compras de importación necesarias para no caer en quiebres, evitando disminuir el nivel de servicio.

Para cada producto se realiza un pronóstico, esto implica un manejo complejo de los datos sin sistemas de apoyo, teniendo en cuenta esta complejidad y dependiendo de la clasificación se determina establecer un modelo específico para ellos, el cual se puede evaluar en periodos cíclicos y así validar que el modelo sea el que más se ajuste a la demanda.

$\mathrm{Al}$ aplicar la etapa cuatro, se toman las directrices de las etapas uno y dos que permiten definir las políticas de inventario a utilizar para cada clasificación, este sentido se observó que la aplicación de la política de revisión periódica para la clasificación A, apuntando al nivel de servicio, genera costos de almacenamiento más altos, pero se aprecia facilidad en la administración [14]. Aun cuando se usaron dos políticas de inventarios distintas se observa que se logró un manejo del sobre stock y/o quiebres de stock.

La Figura 2, expone los niveles de servicio alcanzados después de la aplicación del modelo por un periodo de tres meses, se evidencia que los productos clasificados como A y B superan el nivel de servicio del $98 \%$ definido por la empresa como parte de su estrategia competitiva. Como se aprecia en la Tabla 1, las clasificaciones A y B son las más significativas en relación al nivel de servicio de la

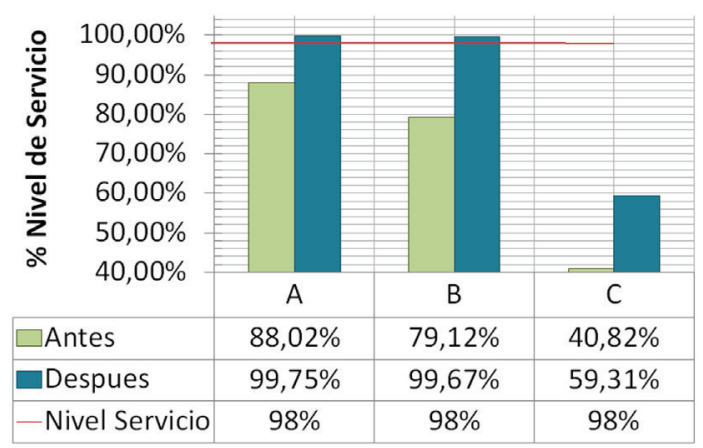

Figura 2. Cambio Niveles de Servicio. empresa con un $95 \%$ de representación, expuesta en la Tabla 3.

Como se puede apreciar en la Tabla 3, el nivel de servicio que se utiliza, es una ponderación de las clasificaciones A, B y C. Este nivel de servicio promedio en tres meses, obtenido bajo el ponderador de clasificación es de un $97,72 \%$. Lo que indica que la implementación del modelo, permite alinear la gestión de inventarios con la estrategia de la empresa.

Los productos clasificados como $\mathrm{C}$, se exponen en la Figura 2 y alcanzaron el 59,31\% de nivel de servicio, observándose un aumento del $18,43 \%$, por tanto, no se alcanza el nivel de servicio definido por la empresa del $98 \%$, pero se estima que el valor del nivel de servicio puede aumentar interpretando la tendencia observada en el comportamiento del nivel de servicio para la clasificación C, como se expone en la Tabla 4 y Figura 3, respectivamente.

Se estima que; si el modelo se mantiene aplicado por un periodo superior a los tres meses, la clasificación C tenderá a alcanzar el nivel de servicio del $98 \%$ definido por la empresa.

El tiempo tomado en alcanzar el nivel de servicio definido, está relacionado por la cantidad de productos que componen la clasificación $\mathrm{C}$, los que son notoriamente superiores al de las clasificaciones A y B como se expone en la Tabla 1.

Como elemento validador de la hipótesis, que indica que; "al aplicar el modelo, el promedio mensual del nivel de servicio tiende al 98\%", se seleccionó un grupo de control.

Tabla 4. Variación nivel servicio clasificación C.

\begin{tabular}{|c|c|c|}
\hline $\begin{array}{c}\text { Aplicación } \\
\text { Modelo }\end{array}$ & Mes-año & $\begin{array}{c}\text { \% Nivel de } \\
\text { Servicio }\end{array}$ \\
\hline No & mar-15 & $20,40 \%$ \\
\hline No & abr-15 & $55,60 \%$ \\
\hline No & may-15 & $17,80 \%$ \\
\hline No & jun-15 & $27,68 \%$ \\
\hline Si & jul-15 & $37,37 \%$ \\
\hline $\mathrm{Si}$ & ago-15 & $58,64 \%$ \\
\hline $\mathrm{Si}$ & sep-15 & $57,34 \%$ \\
\hline $\mathrm{Si}$ & oct-15 & $61,95 \%$ \\
\hline
\end{tabular}




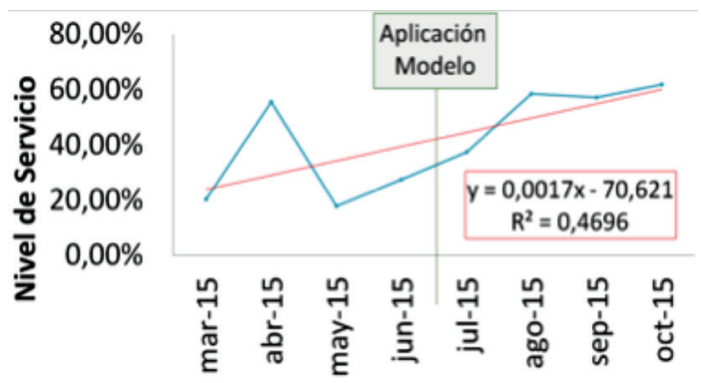

Figura 3. Tendencia clasificación C.

Para determinar la muestra de productos que se utilizarán como grupo de control, se trabaja sobre el universo total de los productos clasificados como A, B y C, expuestos en la Tabla 1, lo que entrega una suma de 11.806 productos.

Teniendo el tamaño de la población de 11.806 en número de producto $(\mathrm{N})$, se establece un error estándar (se) del 0.015 y una probabilidad de ocurrencia del fenómeno (p) de 0,9. Con los datos determinado se calcula la varianza de la muestra, expresada como la probabilidad de ocurrencia y la varianza de la población al cuadrado. Utilizando estos datos se determina que el tamaño de la muestra es de 387 productos.

Para obtener la cantidad de productos que compondrán cada clasificación se determina el factor para la estratificación (ksh) [16] que corresponde a un 0,033 . Multiplicando el factor ksh con cada valor de la columna $\mathrm{N}^{\circ}$ Productos de la Tabla 1 para las clasificaciones A, B y C, se obtiene la cantidad de productos que componen el grupo de control, como se expone en la Tabla 5 en la columna $\mathrm{N}^{\circ}$ Muestras.

El grupo de control, es creado calculando la muestra estadística valida de los productos clasificados A, B y C [14], A los cuales no se les aplico el modelo, el resultado se observa en la Figura 4. Los productos del grupo de control son obtenidos de forma aleatoria de cada clasificación.

Tabla 5. Cantidad de productos del grupo de control.

\begin{tabular}{|c|c|c|}
\hline Clasificación & $\mathbf{N}^{\mathbf{0}}$ Productos & $\mathbf{N}^{\mathbf{o}}$ Muestras \\
\hline $\mathrm{A}$ & 476 & 16 \\
\hline $\mathrm{B}$ & 1.397 & 46 \\
\hline $\mathrm{C}$ & 9.933 & 326 \\
\hline TOTAL & 11.086 & 387 \\
\hline
\end{tabular}

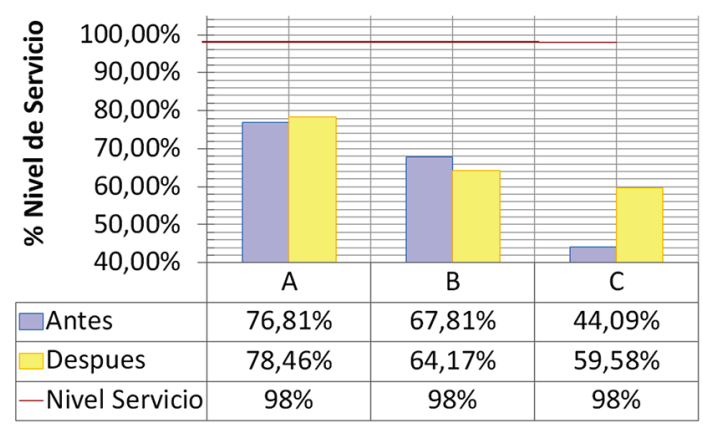

Figura 4. Grupo Control - Cambio Niveles de Servicio.

El grupo de control, no alcanza el nivel de servicio dado por la estrategia de la empresa. Por tanto, la prueba de hipótesis indica que, al aplicar el modelo, el promedio mensual del nivel de servicio tiende de al $98 \%$.

Un elemento no menor a considerar en la aplicación del modelo son las competencias de los colaboradores de la empresa, pues, aportan un rol gravitante en el éxito de la implementación de cada una de las etapas del modelo, pues se requiere de personal capacitado. Para la aplicación de este modelo se contó con personal calificado y capacitado en las respectivas técnicas que el modelo utiliza.

Una vez terminado el proceso de experimentación, todos los productos de la empresa quedaron sujetos a la aplicación del modelo, los resultados para el grupo de control en los años 2016 y 2017 se exponen en la Figura 5, se puede apreciar que el año 2016 en promedio se alcanzó un 97,03\% de nivel de servicio para la clasificación $\mathrm{C}$, esto

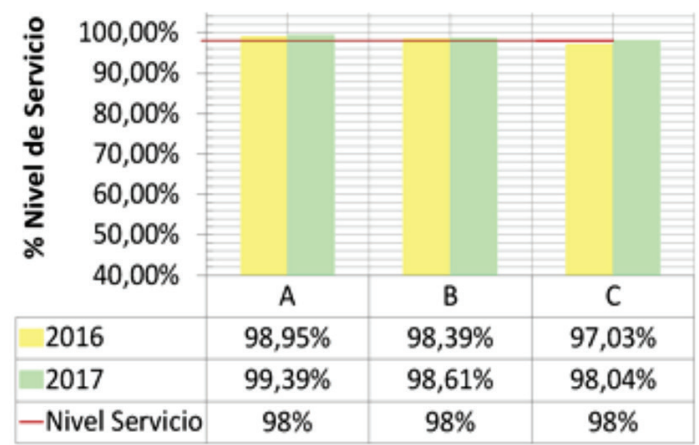

Figura 5. Grupo Control - Niveles de Servicio Promedio para año 2016 y 2017. 
implica que no se alcanzó el nivel de servicio del $98 \%$, esto se relaciona con la cantidad de productos que componen la clasificación C. En el año 2017 se obtuvo un $98,04 \%$ de nivel de servicio logrando llegar al nivel de servicio definido para la empresa.

En relación con el nivel de servicio para todos los productos de las clasificaciones A, B y C, la Figura 6, expone los valores anuales del nivel de servicio para los años 2016 y 2017, exponiendo un comportamiento similar al grupo de control.

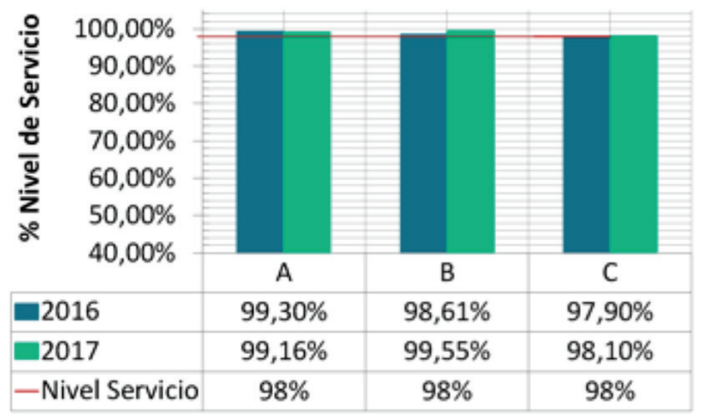

Figura 6. Niveles de Servicio Promedio para año 2016 y 2017.

\section{REFERENCIAS}

[1] G.A. Méndez y E.R. López, "SCIELO", Metodología para el pronóstico de la demanda en ambientes multiproducto y de alta variabilidad, 2708 2013. [En línea]. Available: http://www.scielo.org.co/ scielo.php?script=sci_arttext\&pid=S0123921X2014000200008\&lang=pt. [Último acceso: 1304 2015].

[2] M. Porter, Competitive Advantage: Creating and Sustaining Superior Performance, NY: Free Pr; Edición: Free Press. (1 de junio de 1998), 1998.

[3] A.S. Aguilar, "SCIELO", Un modelo de clasificación de inventarios para incrementar incrementar el nivel de servicio al cliente y la rentabilidad de la empresa, 0103 2012. [En línea]. Available: http://www.scielo.org.co/ scielo.php?script=sci_arttext\&pid=S1657$62762012000100007 \&$ lang=pt. [Último acceso: 1304 2015].

[4] V. Gutiérrez y C.J. Vidal, "Revista facultad de ingeniería, Universidad de Antioquia",
Modelos de Gestión de Inventarios en Cadenas de Abastecimiento: Revisión de la Literatura, 01 2008. [En línea]. Available: http://aprendeenlinea.udea.edu.co/revistas/ index.php/ingenieria/article/view/18765. [Último acceso: 1604 2015].

[5] W.C. Kim y R. Mauborgne, La Estrategia de Océano Azul, Bogotá: Grupo Editorial Norma, 2005.

[6] I. Pérez, A.M. Cifuentes, C. Vásquez y D. Ocampo, "SCIELO", Un modelo de gestión de inventarios para una empresa de productos alimenticios, 01 05 2013. [En línea]. Available: http:// scielo.sld.cu/scielo.php?pid=S181559362013000200011\&script=sci_arttext. [Último acceso: 1504 2015].

[7] D. Anderson y D. Sweeney, Statistics for business and economics, South-Western College Pub: Hardcover, 2008.

[8] M. Valencia Cárdenas, F.J. Díaz Serna y J.C. Correa Morales, "Planeación de inventarios con demanda dinámica. Una revisión del estado del arte", 04 2015. [En línea]. Available: http://www.scielo.org.co/ scielo.php?script=sci_arttext\&pid=S0012$73532015000200023 \&$ lang=pt. [Último acceso: 2505 2015].

[9] L. Krajewski y L. Ritzman, Administración de Operaciones, Estrategia y Análisis, México: PEARSON EDUCACION, 2000.

[10] P. Reyes Aguilar, "Administración de Operaciones - Métodos de Pronósticos", 08 2009. [En línea]. Available: http://www.icicm. com/files/PRONOSTICOS.docx. [Último acceso: 2005 2015].

[11] B. Render, M.R. Stair y M. Hanna, Metodos Cuantitativos para los Negocios, México: Pearson Educación, 2006.

[12] A. Leal, "Universidad del Zulia", Modelo de Planificación y Control de Inventarios para Mantenimiento, 0102 2004. [En línea]. Available: http://tesis.luz.edu.ve/tde_busca/ arquivo.php?codArquivo=1703. [Último acceso: 0405 2015].

[13] M.J. Naranjo de Lucca, "Tesis UChile", manejo de inventarios en una cadena de supermercados, 0212 2006. [En línea]. Available: http://www.tesis.uchile.cl/tesis/ uchile/2006/naranjo_m/sources/naranjo_m. pdf. [Último acceso: 2104 2015]. 
[14] E. Gutiérrez González, O.V. Panteleeva, M.F. Hurtado Ortiz y C. González Navarrete, "SCIELO", Aplicación de un modelo de inventario con revisión periódica para la fabricación de transformadores de distribución, 0201 2013. [En línea]. Available: http://www.scielo.org.mx/pdf/iit/v14n4/ v14n4a7.pdf. [Último acceso: 3004 2015].

[15] L. Kish, Representatividad, aleatorización y realismo, Madrid: CID, 1995.
[16] J.M. Barraza, R. Guiraldes y D. Galindo, Modelo Drivers de Valor para los Negocios y Organizaciones Modernas, Chile: ANTICIPA, 2013.

[17] Ó. Parada. "Un enfoque multicriterio para la toma de decisiones en la gestión de inventarios", 3006 2009. [En línea]. Available: http://www.scielo.org.co/pdf/cadm/ v22n38/v22n38a09.pdf. [Último acceso: 02 05 2015]. 